Pacific Journal of Mathematics

BOOLEAN ALGEBRAS WITH ORDERED BASES

D Dean Mayer and Richard Scott Pierce 


\title{
BOOLEAN ALGEBRAS WITH ORDERED BASES
}

\author{
R. D. Mayer ANd R. S. Pierce
}

1. Introduction. In 1939 Mostowski and Tarski introduced in [6] the notion of a Boolean ring with an ordered basis and they showed that many of the properties of countable Boolean rings can be generalized to this larger class of rings.

Definition 1.1. A subset $S$ of a Boolean ring $B$ is called an ordered basis of $B$ if:

(i) $S$ does not contain the zero 0 of $B$;

(ii) the ordering of $B$ restricted to $S$ is total, that is, for any $s, t$ in $S$, either $s \leq t$ or $t \leq s$;

(iii) $S$ generates $B$, that is, there is no proper subalgebra of $B$ containing $S$.

Not every Boolean ring contains an ordered basis $S$. (See the end of section two.) But a more serious difficulty is the fact that a Boolean ring may have many different ordered bases. Consequently, it seems natural to consider pairs $\langle B, S\rangle$ consisting of Boolean rings $B$ and ordered bases $S \subseteq B$.

In this paper the topological representation theory of Stone [7] is used to study Boolean algebras with ordered bases. We first investigate the topological interpretation of the extra structure provided by an ordered basis in a Boolean algebra. It turns out that the existence of an ordered basis $S$ in $B$ is equivalent to the existence of an ordering $\leq$ of the points of the Boolean space $X$ of $B$ with the property that the topology of $X$ coincides with the interval topology. Moreover, distinct bases in $B$ correspond to different orderings of $X$. Section two is devoted to establishing this correspondence $\langle B, S\rangle \leftrightarrow\langle X, \leq\rangle$ between Boolean algebras with ordered bases and ordered Boolean spaces. The remainder of the paper is devoted to exploiting the correspondence. Boolean algebras with bases of special order types are considered. In particular, a topological characterization of Boolean algebras with well ordered bases is obtained. Moreover, complete invariants for the isomorphism types of such algebras are found and these invariants are used to investigate free product decompositions.

A remark is in order concerning the restriction to Boolean algebras, that is, Boolean rings with a unit 1. From the topological point of view, this restriction yields the blessings of compactness. Moreover,

Received November 10, 1959. This paper is a revision and amplification of part of the first author's doctoral dissertation submitted to the University of Washington. The second named author was supported by the National Science Foundation while working on the paper. 
from the algebraic standpoint the restriction is mild since, as can easily be seen, if $B$ is a Boolean ring with ordered basis $S$, then the Boolean algebra $B_{1}$ obtained from $B$ by the usual process of unit adjunction has $S_{1}=S \cup\{1\}$ as an ordered basis, and what is more, $B$ can be recovered from $\left\langle B_{1}, S_{1}\right\rangle$ as the unique maximal ideal containing $S_{1}-\{1\}$.

It is assumed that the reader of this paper has a working knowledge of the Stone representation theory of Boolean algebras, as developed in [7]. We shall denote by $X(B)$ the Boolean space of prime ideals of the Boolean algebra $B$. Also, for any topological space $X, B(X)$ stands for the Boolean algebra of open-and-closed sets in $X$. Operations in a Boolean algebra are denoted by $\wedge, \vee,\left({ }^{\prime}\right)$, and + (for the symmetric difference). We use small Greek letters to designate ordinal numbers. In particular, $\omega$ denotes the first infinite ordinal. Ordinal numbers are considered as sets, namely the sets consisting of all ordinals less than a given one. Thus, for ordinal numbers, the relations $\alpha<\beta, \alpha \subset \beta$ and $\alpha \in \beta$ are equivalent. We will have occasion to use addition, multiplication, and exponentiation of order types. The definition of these operations are given in [3].

2. Boolean chains. If $x$ and $y$ are elements of a chain and $x<y$, let $(x, y)=\{z \mid x<z<y\},[x, y]=\{z \mid x \leq z \leq y\},(x, y]=\{z \mid x<z \leq y\}$ and $(y]=\{z \mid z \leq y\}$. The element $y$ covers $x$ precisely when $(x, y)=\phi$ (assuming $x<y$ ). In this case it is customary to call $\langle x, y\rangle$ a jump (see [2], p. 90).

Definition 2.1. A chain $C$ has the jump property if for any $x<y$ in $C$, there is a jump in the interval $[x, y]$. An element $t$ in the chain $C$ is called a jump point if either $t$ is covered in $C$ or $t$ is the greatest element of $C$.

Lemma 2.2. Let $C$ be a chain with a greatest element 1 . Let $A$ be the set of all jump points of $C$. Then the following two conditions are equivalent:

(i) $C$ has the jump property;

(ii) the half open intervals $(a, b]$ and $(c]$ with $a, b, c$ in $A$ constitute a base for the interval topology of $C$. If $C$ is complete, then these conditions are all equivalent to $C$ being totally disconnected in its interval topology.

Proof. The equivalence of (i) and (ii) is a routine consequence of the definitions; also, (ii) clearly implies that the open-and-closed sets in $C$ form a basis for the interval topology. If $C$ is complete, then it is compact in its interval topology [1, p. 41]. Assume that $C$ is totally disconnected and let $x<y$ in $C$. We wish to find a jump in the closed 
interval $[x, y]$. If $y$ covers $x$, then $\langle x, y\rangle$ is already a jump. If $x<z<y$ for some $z$, there is an open-and-closed set $N$ with $z \in N \subseteq(x, y)$. By compactness, $N$ is a finite union of non-empty open intervals, say $N=\left(a_{1}, b_{1}\right) \cup \cdots \cup\left(a_{k}, b_{k}\right)$ with $x \leq a_{1} \leq \cdots \leq a_{k} \leq y$. Then $a_{1} \notin N$, so there is an open interval $(c, d)$ containing $a_{1}$ and disjoint from $N$. In particular, $\left(a_{1}, d\right) \subseteq(c, d) \cap\left(a_{1}, b_{1}\right)=\phi$, that is, $d$ covers $a_{1}$. Since $a_{1}<y, x \leq a_{1}<d \leq y$ and $\left\langle a_{1}, d\right\rangle$ is the required jump.

Definition 2.3. A totally ordered set $C$ is called a Boolean chain if $C$ is complete and has the jump property.

A Boolean chain will be considered to be both an ordered set and a topological space with the order topology. By 2.2 and [1, p. 41], an ordered set is a Boolean chain if and only if it is a compact, totally disconnected space in its order topology.

Before formulating the main theorem, it is necessary to establish the notion of isomorphism of Boolean algebras with ordered bases.

Definition 2.4. Let $\langle B, S\rangle$ and $\left\langle B^{\prime}, S^{\prime}\right\rangle$ be pairs consisting of Boolean algebras with ordered bases. A Boolean isomorphism $h$ of $B$ into $B^{\prime}$ is called a basis isomorphism if $h(S)=S^{\prime}$; in this case the pairs $\langle B, S\rangle,\left\langle B^{\prime}, S^{\prime}\right\rangle$ are said to be isomorphic.

As usual, two chains are called isomorphic if there is a one-to-one, order preserving correspondence between them. Such a correspondence automatically preserves the order topology.

Theorem 2.5. (i) Let $B$ be a Boolean algebra and let $S$ be an ordered basis of $B$. Then there is a unique ordering $\leq_{s}$ of $X(B)$ such that

(a) $\left\langle X(B), \leq_{s}\right\rangle$ is a Boolean chain;

(b) the Boolean topology of $X(B)$ coincides with the interval topology determined by $\leq_{s}$;

(c) in the natural isomorphism between $B$ and the open-and-closed sets of $X(B)$, the elements of $S$ correspond to intervals $(J]$, where $J$ is a jump point of $X(B)$.

(ii) Let $\langle C, \leq\rangle$ be a Boolean chain and let $S(\leq)$ be the set of intervals of the form $(a]$, where $a$ is a jump point. Then $S(\leq)$ is an ordered basis for $B(C)$.

(iii) The correspondences $\Sigma:\langle B, S\rangle \rightarrow\left\langle X(B), \leq_{S}\right\rangle$ and $T:\langle C, \leq\rangle \rightarrow$ $\langle B(C), S(\leq)\rangle$ are dual to each other, that is, $T \Sigma\langle B, S\rangle$ is isomorphic to $\langle B, S\rangle$ and $\Sigma T\langle C, \leq\rangle$ is isomorphic to $\langle C, \leq\rangle$.

Proof. (i) Let $P$ and $Q$ be prime ideals of $B$. Define $P \leq_{S} Q$ if $P \cap S \subseteq Q \cap S$. This relation is obviously transitive and reflexive. Moreover, $P \cap S$ and $Q \cap S$ are ideals in the chain $S$, so that either $P \cap S \subseteq Q \cap S$, 
or $Q \cap S \subseteq P \cap S$. Finally, if $P \neq Q$, then $P \cap S \neq Q \cap S$ (see [6, Theorem 4.1]). Thus $\left\langle X(B), \leq_{s}\right\rangle$ is a chain. The fact that this chain is complete and has the jump property will follow from 2.2 once we show that the order topology coincides with the Boolean topology. By the compactness' of $X(B)$ it is enough to prove that every non-empty interval $(P, R)$ is open. Hence, suppose $P<_{S} Q<_{S} R$. We want to find $a \in B$ such that $Q \in X(a) \subseteq(P, R)$, where $X(a)$ is the open-and-closed subset of $X(B)$ corresponding to $a$, namely $X(a)=\{L \in X(B) \mid a \notin L\}$. Since $P \cap S \subset Q \cap S$ $\subset R \cap S$, there exist $t \in Q \cap S-P \cap S$ and $s \in R \cap S-Q \cap S$. Let $a=s \wedge t^{\prime}$. Since $t \in Q, s \notin Q$, it follows that $a \notin Q$, that is, $Q \in X(a)$. If $L \in X(a)$, then $s \wedge t^{\prime} \notin L$, so that $s \notin L$ and $t \in L$. Thus, $L \cap S \nsubseteq P \cap S$ and $R \cap S \nsubseteq L \cap S$. Hence $P<_{S} L<_{S} R$. This proves that $X(a) \subseteq(P, R)$.

To prove (c), suppose that $s \in S$. Then $P \leq_{s} Q \in X(s)$ implies $s \notin Q \cap S \supseteq P \cap S$. so that $P \in X(s)$. Thus, $X(s)$ is an open-and-closed ideal of the chain $X(B)$ and therefore of the form $(J]$, where $J$ is a jump point. Conversely, if $J$ is a jump point, then $(J]$ is an open-andclosed ideal of the chain $X(B)$. In particular, $(J]=X(a)$ for some nonzero $a \in B$. Represent $a$ in the form $a_{k}+a_{k-1}+\cdots+a_{2}+a_{1}$, with $a_{i} \in S$ and $0<a_{k}<a_{k-1}<\cdots<a_{2}<a_{1}$. Since $S$ generates $B$, such a representation exists. By what has just been shown, $X\left(a_{i}\right)=\left(J_{i}\right.$ ] for some $J_{i} \in X(B)$. Clearly, $J_{k}<_{S} J_{k-1}<_{S} \cdots<_{S} J_{2}<_{S} J_{1}$. Then $(J]=$ $\left(J_{2}, J_{1}\right] \cup\left(J_{4}, J_{3}\right] \cup \cdots$, which is possible only if $k=1, J=J_{1}$ and $(J]=$ $\left(J_{1}\right]=X\left(a_{1}\right)$.

(ii) is a routine consequence of 2.2 .

(iii) $\mathrm{By}$ [7], the correspondence $a \rightarrow X(a)$ defines an isomorphism of $B$ onto $B(X(B))$. The assertion that this is a basis isomorphism is just a restatement of (i)(c). Thus, $\langle B, S\rangle$ is isomorphic to $T \Sigma\langle B, S\rangle$. Also, if $C$ is a Boolean chain, the mapping $x \rightarrow P_{x}=\{N \in B(C) \mid x \notin N\}$ defines a homeomorphism of $C$ on $X(B(C))$. If $x \leq y$, and if $N \in S(\leq)$, then $y \in N$ implies $x \in N$. Thus, $P_{x} \cap S(\leq) \subseteq P_{y} \cap S(\leq)$. Hence, $\langle C, \leq\rangle$ is isomorphic to $\Sigma T\langle C, \leq\rangle$.

REMARK. If $\langle B, S\rangle$ and $\left\langle B^{\prime}, S^{\prime}\right\rangle$ are Boolean algebras with ordered bases, and if $h$ is homomorphism of $B$ into $B^{\prime}$ such that $h(S) \subseteq S^{\prime}$, then the continuous mapping of $X\left(B^{\prime}\right)$ into $X(B)$ which the Stone representation theory associates with $h$ can be shown to preserve the orderings $\leq_{s^{\prime}}$ and $\leq_{s}$. Dually, if $g$ is a continuous, order preserving mapping of the Boolean chain $\langle C, \leq\rangle$ into the Boolean chain $\left\langle C^{\prime}, \leq^{\prime}\right\rangle$, then the associated homomorphism of $B\left(C^{\prime}\right)$ into $B(C)$ maps $S\left(\leq^{\prime}\right)$ into $S(\leq)$. Thus, the correspondences $\Sigma$ and $T$ are contravariant functors between the categories of Boolean algebras with ordered bases and Boolean chains (with the notion of homomorphism defined suitably). They are dual in the sense that the isomorphisms of 2.5 (iii) define natural equivalences of $T \Sigma$ and $\Sigma T$ to the identity functors on their respective categories. 
Mostowski and Tarski showed that a Boolean algebra $B$ is uniquely determined up to isomorphism by any of its ordered bases. Specifically, if $S$ and $S^{\prime}$ are bases of the Boolean algebras $B$ and $B^{\prime}$ respectively, and if $h$ is a one-to-one, order preserving mapping of $S$ onto $S^{\prime}$, then $h$ extends uniquely to an isomorphism of $B$ onto $B^{\prime}$ [6, Theorem 2.1]. Thus, the ordered basis $S$ of $B$ also determines $\left\langle X(B), \leq_{S}\right\rangle$. However, $X(B)$ can be constructed explicitly from $S$.

If $S$ is a chain, a (possibly empty) subset $I \subseteq S$ is called an ideal of $S$ if $a \in I, b \leq a$ implies $b \in I$. Any element $a \in S$ naturally determines two ideals:

$$
\begin{gathered}
I_{a}=\{x \in S \mid x<a\}, \\
J_{a}=\{x \in S \mid x \leqq a\},
\end{gathered}
$$

Definition 2.6. Let $S$ be a chain with a greatest element 1. Define $c(S)$ to be the set of all ideals $I$ of $S$ such that $1 \notin I$.

LEMma 2.7. ( i ) $c(S)$ is a Boolean chain under inclusion;

(ii) $a \rightarrow I_{a}$ is a one-to-one, order preserving mapping of $S$ onto the set of all jump points of $c(S)$;

(iii) if $S$ is complete, then $c(S)=\left\{I_{a} \mid a \in S\right\} \cup\left\{J_{a} \mid a \in S, a \neq 1\right\}$.

Proof. It is well known that the ideals of a chain form a complete chain under inclusion. From this, it follows easily that $c(S)$ is a complete chain. Moreover, it is clear that for any $a \in S, J_{a}$ covers $I_{a}$ in $c(S)$. If $I \subset J$ are ideals in $c(S)$, and if $a \in J$ and $a \notin I$, then

$$
I \subseteq I_{a} \subset J_{a} \subseteq J
$$

Thus, $c(S)$ has the jump property. Moreover, if $J$ covers $I$, then necessarily $I=I_{a}$ and $J=J_{a}$. Hence every jump point of $c(S)$ is of the form $I_{a}$ for some $a \in S$. (Note that the greatest element of $c(S)$ is $I_{1}$.) This proves (i) and (ii). If $S$ is complete and $I \in c(S)$, let a be the least upper bound of $I$. Then $b \in I$ implies $b \leq a$. Hence $I \subseteq J_{a}$. On the other hand, if $b<a$, then $b \leq c$ for some $c \in I$ and therefore $b \in I$. Thus, $I_{a} \subseteq I$. Consequently, either $I=I_{a}$, or $I=J_{a}$. This proves (iii).

THEOREM 2.8. If $S$ is an ordered basis of the Boolean algebra $B$, then $\left\langle X(B), \leq_{s}\right\rangle$ is order isomorphic to $\langle c(S), \subseteq\rangle$.

Proof. The theorem follows from 2.7, 2.5 and the uniqueness theorem of Mostowski and Tarski [6, Theorem 2.1].

${ }^{2} \mathrm{We}$ are indebted to the referee for a suggestion which simplified our original construction of $X(B)$. 
CoRollaRy 2.9. A Boolean algebra $B$ has a well ordered basis if and only if $X(B)$ is homeomorphic to $\kappa$, where $\kappa$ is a non-limit ordinal with the interval topology.

Proof. If $B$ has a well ordered basis $S$, then $S$ is order isomorphic to some ordinal $\kappa$. Since $S$ has a greatest element, $\kappa$ is a non-limit ordinal. Since $\kappa$ is complete and all of its points except the greatest are covered, $c(\kappa)=\kappa$. Hence, $X(B)$ is homeomorphic to $\kappa$ by 2.8. The converse is a consequence of 2.5.

We conclude this section with a result which provides examples of Boolean algebras having no ordered basis.

THEOREM 2.10. If $B$ is an infinite Boolean algebra with an ordered basis $S$, then $B$ is not countably complete.

Proof. Clearly $S$ is infinite. Suppose $S$ contains no descending chain of type $\omega^{*}$. Then $S$ is well ordered and therefore contains a subset of type $\omega$. Hence $S$ has either an infinite descending or an

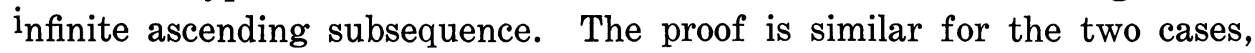
so assume that $S$ contains a sequence $s_{1}<s_{2}<\ldots$ By 2.5, $X\left(s_{n}\right)=$ $\left(J_{n}\right]$, where $J_{n}$ is covered in $X(B)$. Then $J_{2 n} \in\left(J_{2 n-1}, J_{2 n}\right]=X\left(s_{2 n-1}+s_{2 n}\right)$. Suppose $t=\bigvee_{n=1}^{\infty}\left(s_{2 n-1}+s_{2 n}\right)$ exists. Then $X(t)$ is a finite union of intervals and one of these necessarily contains a pair of points $J_{2 m}, J_{2 n}$ with $m<n$. Hence, $X\left(s_{2 m}+s_{2 m+1}\right) \subseteq\left(J_{2 m}, J_{2 n}\right] \subseteq X(t)$ and therefore $s_{2 m}+s_{2 m+1} \leq t$. But clearly $\left(s_{2 m}+s_{2 m+1}\right) \wedge t=0$, so $s_{2 m}=s_{2 m+1}$. This contradiction proves the theorem.

3. Boolean algebras with scattered bases. A totally ordered set is called scattered if it contains no subset whose order type is the same as that of the rational numbers. For example, any well ordered set is scattered. In this section we will study those Boolean algebras which have a scattered basis, that is, an ordered basis which is scattered. The first objective is to characterize the Boolean spaces of these algebras.

Definition 3.1. Let $X$ be any Hausdorff topological space. Define $X^{\prime}$ to be the subspace of $X$ consisting of all limit points, that is, the complement of the set of isolated points of $X$. The space $X^{\prime}$ is called the (first) derivative of $X$. Transfinite derivatives of $X$ are defined inductively: $X^{(0)}=X ; \quad X^{(\tau+1)}=\left(X^{(\tau)}\right)^{\prime} ; \quad X^{(\sigma)}=\bigcap_{\tau<\sigma} X^{(\tau)}$, when $\sigma$ is a limit ordinal.

It is convenient to collect some properties of the transfinite derivatives.

LEMma 3.2. If $X$ is a Hausdorff space and $\sigma$ and $\tau$ are any 
ordinals, then

(i) $\sigma>\tau$ implies $X^{(\sigma)} \subseteq X^{(\tau)}$;

(ii) for some smallest ordinal $\tau, X^{(\tau)}=X^{(\tau+1)}$; for any larger $\sigma, X^{(\tau)}=X^{(\sigma)}$

(iii) $X^{(\tau)}$ is a closed subspace of $X$;

(iv) $X \supseteq Y$ implies $X^{(\tau)} \supseteq Y^{(\tau)}$ :

(v) $\left(X^{(\sigma)}\right)^{(\tau)}=X^{(\sigma+\tau)}$;

(vi) if $Y$ is an open subset of $X$, then $Y^{(\tau)}=Y \cap X^{(\tau)}$;

(vii) if $X$ is compact and $X^{(\tau)}=\phi$, then there is a non-limit ordinal $\lambda$ such that $X^{(\lambda)}=\phi$ and $X^{(\lambda-1)}$ is a finite, non-empty set:

(viii) if $X$ is a chain and $X^{(\tau)}=\phi$ for some $\tau$, then $X$ has the jump property.

Proof. Properties (i)-(v) are simple consequences of the definitions. To prove (vi), note that by (i) and (iv), $Y^{\prime} \subseteq Y \cap X^{\prime}$. Suppose $x \in Y$ and $x \notin Y^{\prime}$. Then there is a neighborhood $N$ of $x$ such that $N \cap Y=\{x\}$. If $Y$ is open, $N \cap Y$ is open and therefore $x \notin X^{\prime}$. Thus, $Y^{\prime}=Y \cap X^{\prime}$. Assume that (vi) holds for all $\xi<\tau$. If $\tau=0, Y^{(0)}=Y=Y \cap X=$ $Y \cap X^{(0)}$. If $\tau=\xi+1$, then since $Y \cap X^{(\xi)}$ is an open subset of $X^{(\xi)}$, $Y^{(\tau)}=\left(Y^{(\xi)}\right)^{\prime}=\left(Y \cap X^{(\xi)}\right)^{\prime}=Y \cap X^{(\xi)} \cap\left(X^{(\xi)}\right)^{\prime}=Y \cap X^{(\tau)}$. If $\tau$ is a limit ordinal, $Y^{(\tau)}=\bigcap_{\xi<\tau} Y^{(\xi)}=\bigcap_{\xi<\tau}\left(Y \cap X^{(\xi)}\right)=Y \cap X^{(\tau)}$. If $X$ is compact and infinite, then $X^{\prime} \neq \phi$. Also, if $\tau$ is a limit ordinal and $X^{(\xi)} \neq \phi$ for all $\xi<\tau$, then $X^{(\tau)} \neq \phi$. From these two remarks, (vii) is evident. Suppose that $X$ is a chain and $(x, y)$ is a non-empty open interval of $X$. Then by (vi), $(x, y)^{(\tau)}=(x, y) \cap X^{(\tau)}=\phi$. In particular, $(x, y)^{\prime} \neq(x, y)$, which clearly implies that $(x, y)$ contains a jump.

THEOREM 3.3. For a Boolean algebra $B$ with an ordered basis $S$, the following properties are equivalent:

(i) every ordered basis of $B$ is scattered;

(ii) $S$ is scattered;

(iii) no quotient algebra of $B$ is atom free;

(iv) every closed non-empty subspace of $X(B)$ has isolated points;

(v) there is an ordinal $\tau$ such that $X(B)^{(\tau)}=\phi$.

Proof. The equivalence of (i), (ii) and (iii) is proved in [6, Theorem 3.12 and Theorem 3.15]. Properties (iii) and (iv) are equivalent since, in the duality between Boolean algebras and Boolean spaces, quotient algebras correspond to closed subspaces and atoms correspond to isolated points. We shall not give this argument in detail. To prove that (iv) and (v) are equivalent, suppose $X(B)$ contains no closed non-empty subspaces without isolated points. Let $\tau$ be an ordinal such that $X(B)^{(\tau)}=$ $X(B)^{(\tau+1)}$. Then $X(B)^{(\tau)}$ is a closed subspace of $X(B)$ which has no isolated points. Thus it must be empty. Conversely, if $X(B)$ contains a closed 
non-empty subspace $Y$ without isolated points, then for all $\tau, X(B)^{(\tau)} \supseteq$ $Y^{(\tau)}=Y \neq \phi$.

It is a known fact that a countable Boolean algebra with a scattered basis has a well ordered basis (see [5]). By 3.3, $B$ can have a well ordered basis only if every one of its bases is scattered. But there are Boolean algebras with scattered bases which have no well ordered bases. Our objective in the remainder of this section is to characterize topologically the Boolean algebras with scattered bases which have well ordered bases.

Lemma 3.4. Let $\alpha$ and $\beta$ be infinite limit ordinals and let $C=$ $\alpha+1+\beta^{*}$. Assume that either $\alpha$ or $\beta$ has no countable cofinal subset. Then $B(C)$ is a Boolean algebra with a scattered basis, but $B(C)$ has no well ordered basis.

Proof. $C$ is a complete chain with the jump property and by 2.5 $B(C)$ has an ordered basis which has the same order type as a subchain of $C$ (to be precise, of type $\alpha+\beta^{*}$ ). Clearly any subchain of $C$ is scattered. Thus, $B(C)$ has a scattered basis. Suppose $B(C)$ has a well ordered basis. Then by 2.8 there is a homeomorphism $\varphi$ of $C$ onto some non-limit ordinal $\kappa$. Let $p$ be the point of $C$ between the upper and lower intervals $\alpha$ and $\beta^{*}$. Since $\alpha$ and $\beta$ are limit ordinals and one of them has no countable cofinal subset, $C$ cannot satisfy the first countability axiom at $p$. Thus $\varphi(p)=\mu$, where $\mu$ is a limit ordinal in $\kappa$ with no countable cofinal subset. The upper and lower intervals $\alpha$ and $\beta^{*}$ of $C$ have the property that the intersection of their closures is $p$. Thus $\varphi(\alpha)^{-} \cap \varphi\left(\beta^{*}\right)^{-}=\{\mu\}$. But this is impossible. For it implies that if $\xi<\mu$, then $\varphi(\alpha) \cap(\xi, \mu) \neq \phi$ and $\varphi\left(\beta^{*}\right) \cap(\xi, \mu) \neq \phi$. Thus, sequences $\left\{x_{1}, x_{2}, \cdots\right\} \subseteq \varphi(\alpha),\left\{y_{1}, y_{2}, \cdots\right\} \subseteq \varphi\left(\beta^{*}\right)$ can be chosen so that $x_{1}<$ $y_{1}<x_{2}<y_{2}<\cdots<\mu$. Let $z=\sup x_{n}=\sup y_{n}$. Then $z \in \phi(\alpha)^{-} \cap \varphi\left(\beta^{*}\right)^{-}=$ $\{\mu\}$, contrary to the non-existence of countable cofinal subsets of $\mu$.

If $C$ is a chain and $x \in C$, the character $\left\langle\rho, \sigma^{*}\right\rangle$ of $x$ (in $C$ ) is defined by the conditions:

(i) $\rho=1$ if $x$ covers or is the least element of $C$;

(ii) $\rho=\omega_{\alpha}$ if $\{y \in C \mid y<x\}$ is cofinal with the regular ordinal $\omega_{\alpha}$;

(iii) $\sigma=1$ if $x$ is covered or is the greatest element of $C ; \sigma=\omega_{\beta}$ if $\{y \in C \mid y>x\}$ is coinitial with $\omega_{\beta}^{*}$, where $\omega_{\beta}$ is regular. These conditions uniquely determine $\rho$ and $\sigma$ (see [2], p. 142).

We shall now show how the example 3.4 leads to a restriction on the possible characters of spaces which are homeomorphic to non-limit ordinals. Two simple facts are needed.

Lemma 3.5. Let $W$ be a well ordered subset of a chain C. Suppose the order type of $W$ is the ordinal $\kappa$. Then the closure of $W$ in $C$ 
has order type either $\kappa$ or $\kappa+1$.

Proof. Assume inductively that the lemma is true for well ordered subsets of $C$ which are of type less than $\kappa$. If $\kappa=\xi+1$, then $W$ has a largest point $p$ and $W-\{p\}$ has order type $\xi$. Hence $W^{-}=(W-$ $\{p\})^{-} \cup\{p\}$ has order type the same as, or one greater than the order type of $(W-\{p\})^{-}$, depending on whether $p \in(W-\{p\})^{-}$, or $p \notin(W-$ $\{p\})^{-}$. By the induction hypothesis $(W-\{p\})^{-}$has order type $\xi$ or $\xi+1=\kappa$. If the order type is $\xi$, then $p \notin(W-\{p\})^{-}$, since otherwise the order type of $W^{-}$would be less than the order type of $W$. Thus if $\kappa$ is a non-limit ordinal, the induction hypothesis leads easily to the desired conclusion. Suppose therefore that $\kappa$ is a limit ordinal. Let $\phi$ be the (unique) one-to-one, order preserving mapping of $\kappa$ onto $W$. For $\xi<\kappa$, let $W_{\xi}=\varphi(\xi)$ (where $\xi$ is considered as a subset of $\kappa$ ). Then $W_{\xi}$ is an initial segment of $W$ and $W_{\xi}$ has order type $\xi$. Hence, $W_{\xi}^{-}$ is an initial segment of $W^{-}$. By the induction hypothesis, there exist one-to-one, order preserving mapping $\psi_{\xi}$ of $W_{\xi}^{-}$onto an initial segment of $\kappa$ for all $\xi<\kappa$. If $\xi<\eta<\kappa$, then $W_{\xi}^{-}$is an initial segment of $W_{\eta}^{-}$, so $\psi_{\eta}$ maps $W_{\bar{\xi}}^{-}$onto an initial segment of $\kappa$. By uniqueness $[1, \mathrm{p} .35]$, this implies $\psi_{\eta} \mid W_{\xi}^{-}=\psi_{\xi}$. Thus, the union $\psi$ of all $\psi_{\xi}$ 's is a well defined, one-to-one, order preserving mapping of $\bigcup_{\xi<\kappa} W_{\xi}^{-}$onto $\kappa$. But $\bigcup_{\xi<\kappa} W_{\xi}^{-}$is an initial segment of $W^{-}$and this initial segment contains $W$. Thus, either $\bigcup_{\xi<\kappa} W_{\xi}^{-}=W^{-}$, or $W^{-}$consists of $\bigcup_{\xi<\kappa} W_{\xi}^{-}$and a single point $p$ greater than all points in this union. In these respective cases, $W^{-}$has order type $\kappa$ and $\kappa+1$.

The next result is well known, so we omit its proof.

Lemma 3.6. If $C$ is a complete chain and $A$ is a closed subset of $C$, then the relative topology of $A$ as a subspace of $C$ is the same as its order topology.

LEMma 3.7. Let $C$ be a complete chain which is homeomorphic to an ordinal. Then each point in $C$ has character of one of the following types: $\langle 1,1\rangle,\langle\rho, 1\rangle,\left\langle 1, \sigma^{*}\right\rangle,\left\langle\omega, \omega^{*}\right\rangle$.

Proof. Suppose some point of $C$ has character $\left\langle\omega_{\alpha}, \omega_{\beta}^{*}\right\rangle$, where either $\alpha>0$ or $\beta>0$. By 3.5, $C$ contains a closed subset $A$ of order type $\omega_{\alpha}+1+\omega_{\beta}^{*}$, where either $\omega_{\alpha}$ or $\omega_{\beta}$ has no countable cofinal subset (because of the regularity of $\omega_{\alpha}$ and $\omega_{\beta}$ ). Under the homeomorphism of $C$ onto an ordinal, $A$ is mapped into a closed subset. Thus, by 3.6, $\omega_{\alpha}+1+\omega_{\beta}^{*}$ is homeomorphic to a well ordered chain, contrary to 3.4 .

One more property is needed for the characterization of complete chains which are homeomorphic to ordinals. A transfinite sequence $\left\{x_{\xi} \mid \xi<\rho\right\}$ (where $\rho$ is a limit ordinal) will be called continuous if 
$\lim _{\xi<\eta} x_{\xi}=x_{\eta}$ for all limit ordinals $\eta<\rho$. In particular, any sequence of type $\omega$ is continuous.

Lemma 3.8. Let $C$ be a complete chain which is homeomorphic to an ordinal. Let $p \in C$ have the character $\langle\rho, 1\rangle$, with $\rho$ infinite. Then there is a continuous increasing sequence $S$ of type $\rho$ in $C$, converging to $p$, and such that every element of $S$ is covered in $C$.

Proof. Let $\varphi$ be a homeomorphism of $C$ onto the non-limit ordinal $\kappa$. Suppose $\varphi(p)=\mu<\kappa$. Then $\mu$ is a limit ordinal, since $p$ is not isolated in $C$. Since $\phi$ is a homeomorphism and $p$ is covered in $C$, there is an ordinal $\nu<\mu$ such that $(\nu, \mu] \subseteq \varphi((p])$. For each $\eta \in(\nu, \mu]$, let $y_{\eta}=\sup \varphi^{-1}((\nu, \eta])$. Since $(\nu, \eta]$ is open-and-closed, $y_{\eta}$ is the largest element of $\phi^{-1}((\nu, \eta]), y_{\eta}$ is covered in $C$, and $y_{\eta} \leq p$ with equality only if $\eta=\mu$. If $\xi$ is a limit ordinal $\leq \mu$, then $y_{\xi}=\sup \Phi^{-1}((\nu, \xi])=$ $\sup \varphi^{-1}\left(\left(\mathbf{U}_{\eta<\xi}(\nu, \eta]\right)^{-}\right)=\sup \left(\left(\mathbf{U}_{\eta<\xi} \Phi^{-1}((\nu, \eta])\right)^{-}\right)=\sup \left(\mathbf{U}_{\eta<\xi} \Phi^{-1}((\nu, \eta])\right)=$ $\sup _{\eta<\xi} y_{\eta}$. Consequently, $Y=\left\{y_{\eta} \mid \nu<\eta \leq \mu\right\}$ is closed in $C$ and $p \in Y^{\prime}$. Thus, $Y$ contains a continuous, increasing sequence $S$ of type $\rho$ converging to $p$. Since every element of $Y$ is covered in $C, S$ has the same property.

REMARK. It is clear from 3.5 that in any complete chain $C$ any point of character $\langle\rho, 1\rangle$ is the limit of a continuous sequence of type $\rho$, but the points of this sequence need not be covered in $C$. On the other hand, in a chain with the jump property any point of character $\langle\rho, 1\rangle$ is the limit of an increasing transfinite sequence, every element of which is covered, but the sequence need not be continuous. For example, in the chain $\left(1+\omega^{*}\right) \omega_{1}+1$, the greatest element has the character $\left\langle\omega_{1}, 1\right\rangle$, but it is clearly not the limit of continuous sequence, all points of which are covered. Thus by 3.8, this chain cannot be homeomorphic to an ordinal. ${ }^{3}$

ThEOREM 3.9. Let $C$ be a complete chain. Then $C$ is homeomorphic to the Boolean space of a Boolean algebra with a well ordered basis if and only if

(i) $C^{(\tau)}=\phi$ for some ordinal $\tau$;

(ii) every interior point of $C$ either covers, is covered, or has the character $\left\langle\omega, \omega^{*}\right\rangle$;

(iii) every point in $C$ which has character $\langle\rho, 1\rangle$ with $\rho$ infinite is the limit of a continuous, increasing sequence of type $\rho$, consisting of elements covered in $C$, and dually for points having character $\left\langle 1, \sigma^{*}\right\rangle$.

The conditions (i), (ii) and (iii) are necessary for $C$ to be homeomorphic

3 Professor J. R. Isbell has shown us a somewhat different proof of this fact, and his idea influenced the development of 3.8. We are indebted to him for his helpful suggestion. 
to an ordinal by $3.3,3.7$ and 3.8 . The proof that these conditions are sufficient will follow a lemma.

LemMA 3.10. Let $C$ be a compact Hausdorff space and suppose there is a continuous mapping $\rho$ of $C$ onto an ordinal number $\lambda$ such that

(i) $\varphi^{-1}(\xi)$ is homeomorphic to an ordinal $\alpha_{\xi}$ for all $\xi<\lambda$;

(ii) $\alpha_{\xi}=1$ if $\xi$ is a limit ordinal.

Then $C$ is homeomorphic to an ordinal number.

Proof. Define $\kappa=\sum_{\xi<\lambda} \alpha_{\xi}$. Suppose that for each $\xi$ in $\lambda, \psi_{\xi}$ is a homeomorphism of $A_{\xi}=\mathcal{P}^{-1}(\xi)$ onto $\alpha_{\xi}$. Define $\chi: C \rightarrow \kappa$ by

$$
\chi(x)=\sum_{\eta<\xi} \alpha_{\eta}+\psi_{\xi}(x), \text { for } x \in A_{\xi} .
$$

Since $C$ is the disjoint union of the sets $A_{\xi}, \chi$ is unambiguously defined for all $x \in C$. Because each $\psi_{\xi}$ is one-to-one and onto, $\chi$ has the same property. In view of the compactness of $C$, it only remains to show that $\chi$ is continuous.

Let $\mu \in \kappa$, say $\mu=\chi(x)$. Suppose $x \in A_{\xi}$. Then $\mu=\sum_{\eta<\xi} \alpha_{\eta}+\psi_{\xi}(x)$. If $\xi$ is a non-limit ordinal, then the intervals of the form $I=\left[\sum_{\eta<\xi} \alpha_{\eta}+\right.$ $\left.\rho, \sum_{\eta<\xi} \alpha_{\eta}+\psi_{\xi}(x)\right]$, where $\rho \leq \psi_{\xi}(x)$ is a non-limit number, form a neighborhood basis of $\mu$ in $\kappa$. Moreover, $\chi^{-1}(I)=\psi_{\xi}^{-1}([\rho, \psi(x)])$ is open in $A_{\xi}$. But $A_{\xi}$ is open-and-closed because $\phi$ is continuous and $\xi$ is isolated. Thus, $\chi^{-1}(I)$ is open. If $\xi$ is a limit ordinal, then $\alpha_{\xi}=1$ and the intervals $I=\left[\sum_{\eta \leqq \zeta} \alpha_{\eta}, \sum_{\eta \leqq \xi} \alpha_{\eta}\right]$, where $\zeta<\xi$, constitute a neighborhood basis of $\mu$. Moreover, $\chi^{-1}(I)=\phi^{-1}((\zeta, \xi])$ is open in $C$. (Note that every $\alpha_{\eta}$ is a non-limit number and therefore $\sum_{\eta \leqq \zeta} \alpha_{\eta}$ is a non-limit number.)

Proof of 3.9. By 3.2 (vii), there is a non-limit ordinal $\lambda$ such that $X(B)^{(\lambda-1)}$ is finite. We prove by induction on $\lambda$ that $C$ is homeomorphic to an ordinal number. If $\lambda=1$, then $C$ is finite and homeomorphic to an ordinal. Assume that $\lambda>1$ and the theorem holds for all chains $\bar{C}$ such that $\bar{C}^{(\lambda-1)}=\phi$. We can also assume that $C^{(\lambda-1)}$ consists of a single point $p$. For by the jump property and 3.2 (vi), $C$ is a disjoint union $C_{1} \cup \cdots \cup C_{n}$ of open-and-closed intervals such that $C_{i}^{(\lambda-1)}$ contains only one point. Clearly each $C_{i}$ satisfies (ii) and (iii) of 3.9. If $C_{i}$ is homeomorphic to $\kappa_{i}$, then $C$ is homeomorphic to $\kappa_{1}+\cdots+\kappa_{n}$. The proof is completed by considering the possible characters of $p$, subject to condition (ii).

Case 1. The character of $p$ is $\left\langle\omega, \omega^{*}\right\rangle$. Then in $C$ there exists a strictly increasing sequence $x_{1}<x_{2}<\cdots<p$ and a strictly decreasing 
sequence $z_{1}>z_{2}>\cdots>p$ such that $\lim x_{n}=\lim z_{n}=p$. Because of the jump property, we can assume that each $x_{n}$ is covered by a point $y_{n}$ and each $z_{n}$ covers a point $w_{n}$. Define the open-and-closed intervals $D_{0}=\left(x_{1}\right], D_{n}=\left[y_{n}, x_{n+1}\right], E_{0}=\left[z_{1}, 1\right], E_{n}=\left[z_{n+1}, w_{n}\right]$. These sets are pairwise disjoint and their union is $C-\{p\}$. Moreover, $D_{n}^{(\lambda-1)}=E_{n}^{(\lambda-1)}=\phi$ for all $n$ by 3.2 (vi). Hence, by the induction hypothesis, each $D_{n}$ and $E_{n}$ is homeomorphic to an ordinal. Define $\varphi: C \rightarrow \omega+1$ by $\varphi(x)=2 n$ if $x \in D_{n}, \varphi(x)=2 n+1$ if $x \in E_{n}$, and $\varphi(p)=\omega$. It is clear that $\phi$ is continuous and $\varphi^{-1}(2 n)=D_{n}, \varphi^{-1}(2 n+1)=E_{n}$. Thus, 3.10 applies and $C$ is homeomorphic to an ordinal number.

Case 2. The character of $p$ is $\langle\rho, 1\rangle$ or $\left\langle 1, \sigma^{*}\right\rangle$. We can assume that the character is $\langle\rho, 1\rangle$, since the second possibility is transformed into the first if $C$ is replaced by $C^{*}$. Since $\lambda>1, \rho$ is infinite. Thus, by property (iii), there is a continuous increasing sequence $S=\left\{x_{\xi} \mid \xi<\rho\right\}$ such that each $x_{\xi}$ is covered and $\lim _{\xi<\rho} x_{\xi}=p$. Define $\varphi(x)=0$ if $x \leq x_{0}, \varphi(x)=\xi$ if $\xi$ is a non-limit number $<\rho$ and $x_{\xi-1}<x \leq x_{\xi}, \varphi\left(x_{\xi}\right)=\xi$ if $\xi$ is a limit number $\langle\rho, \varphi(p)=\rho$, and $\varphi(x)=\rho+1$ if $x>p$. Using the fact that $S$ is continuous and the points of $S$ are covered, it is easy to see that $\varphi(x)$ is defined for all $x$ in $C$ and $\phi$ is continuous. If $\xi$ is a non-limit number $<\rho$, then $\varphi^{-1}(\xi)=\left(x_{\xi-1}, x_{\xi}\right]$ is an open-and-closed interval of $C$ satisfying $\left(x_{\xi-1}, x_{\xi}\right]^{(\lambda-1)}=\phi$. Hence, by the induction hypothesis $\varphi^{-1}(\xi)$ is homeomorphic to an ordinal number. Similarly $\varphi^{-1}(\rho+1)$ is homeomorphic to an ordinal number. Finally, by $3.10, C$ is homeomorphic to an ordinal number.

Using 2.8 , it is possible to translate 3.9 into an algebraic condition for a Boolean algebra with an ordered basis to have a well ordered basis. However, we shall be content to give two sufficient conditions for the existence of a well ordered basis.

CoRollary 3.11 (Mazurkiewicz-Sierpinski). Any countable Boolean algebra with a scattered basis has a well ordered basis.

Proof. If $S$ is a scattered basis of $B$, then $\left\langle X(B), \leq_{S}\right\rangle$ satisfies the first countability axiom, so the conditions (ii) and (iii) are fulfilled.

Corollary 3.12. If $B$ is a Boolean algebra with a complete, scattered basis, then $B$ has a well ordered basis.

Proof. If $S$ is a complete scattered basis of $B$, then the chain $\left\langle X(B), \leq_{s}\right\rangle$ is complete, has some transfinite derivative empty (by 3.3), and every interior point of this chain either covers or is covered (by 2.7 and 2.8). If $p \in X(B)$ has character $\langle\rho, 1\rangle$, let $S$ be an increasing sequence of type $\rho$ approaching $p$, such that every point of $S$ is covered. 
By 3.5, $S^{-}-\{p\}$ is a continuous increasing sequence of type $\rho$ approaching $p$. If $x \in S^{-}-S$, then $x$ is the limit of an increasing segment of $S$. In particular, $x$ does not cover. But then $x$ is covered in $C$. Similarly, every point of $X(B)$ of character $\left\langle 1, \sigma^{*}\right\rangle$ is the limit of a decreasing, continuous sequence of type $\sigma^{*}$, all elements of which cover.

4. Boolean algebras with well ordered bases. The object of this section is to show that a Boolean algebra with a well ordered basis has a canonical basis. We restrict our attention to infinite Boolean algebras, since the finite case is trivial.

Lemma 4.1. If $\beta$ and $\gamma$ are ordinal numbers, then the Boolean chains $\beta+\gamma+1$ and $\gamma+\beta+1$ are homeomorphic.

Proof. The space $\beta+\gamma+1$ is the disjoint union of the open-andclosed intervals $(0],[1, \beta],[\beta+1, \beta+\gamma]$ and $[\beta+1, \beta+\gamma]$ is homeomorphic to $[1, \gamma]$. Similarly, $\gamma+\beta+1$ is homeomorphic to the disjoint union of these same intervals.

THEOREM 4.2. Let $\beta$ be an infinite Boolean algebra with a well ordered basis. Then $B$ has a basis of order type $\omega^{\alpha} \cdot n+1$, where $\alpha$ is an ordinal $>0$ and $n$ is a positive integer.

Proof. By 2.9, $X(B)$ is homeomorphic to $\kappa+1$ for some infinite ordinal number $\kappa$. Let $\kappa=\omega^{\alpha} n+\gamma$, with $n>0$ and $\gamma<\omega^{\alpha}$ [3, p. 67]. By 4.1, $X(B)$ is homeomorphic to $\gamma+\omega^{\alpha} \cdot n+1=\omega^{\alpha} n+1$ [3, p. 68]. Thus $B$ has an ordered basis of type $\omega^{\alpha} \cdot n+1$ by 2.5 .

The remainder of this section is devoted to proving that the pair $\langle\alpha, n\rangle$ in 4.2 is an invariant of $B$. This result is a simple generalization of Theorem 1 in [5]. The idea of the proof (due to Mazurkiewicz and Sierpenski) is simple. If $B$ has an ordered basis of type $\omega^{\alpha} \cdot n+1$, then $X(B)$ is homeomorphic to the Boolean chain $\omega^{\alpha} \cdot n+1$. What we prove is that if $\alpha>0,\left(\omega^{\alpha} \cdot n+1\right)^{(\alpha)}$ contains precisely $n$ points. This topological property must be shared by $X(B)$. Therefore the algebraic structure of $B$ determines both $n$ and $\alpha$.

Lemma 4.3. Let $\gamma, \kappa$ and $\tau$ be ordinals with $\gamma<\kappa$. Then $\gamma \in \kappa^{(\tau)}$ if and only if $\gamma \in(\gamma+1)^{(\tau)}$.

This is obvious.

Lemma 4.4. Let $\beta, \gamma, \kappa$ and $\tau$ be ordinals with $\gamma>0$ and $\beta+\gamma<\kappa$. Then $\beta+\gamma \in \kappa^{(\tau)}$ if and only if $\gamma \in \kappa^{(\tau)}$.

Proof. Let $M=[1, \gamma]$ and $N=[\beta+1, \beta+\gamma]$. Then $M$ and $N$ 
are open intervals in $\kappa$ and the mapping $\xi \rightarrow \beta+\xi$ is a homeomorphism of $M$ on $N$ sending $\gamma$ onto $\beta+\gamma$. Thus, by 3.2 (vi), $\gamma \in \kappa^{(\tau)}$ if and only if $\gamma \in M \cap \kappa^{(\tau)}=M^{(\tau)}$ and $\gamma \in M^{(\tau)}$ is equivalent to $\beta+\gamma \in N^{(\tau)}=N \cap \kappa^{(\tau)}$. Hence, $\gamma \in \kappa^{(\tau)}$ if and only if $\beta+\gamma \in \kappa^{(\tau)}$.

\section{Lemma 4.5. If $\alpha>0$, then $\left(\omega^{\alpha}+1\right)^{(\alpha)}=\left\{\omega^{\alpha}\right\}$.}

Proof. This is clear if $\alpha=1$. Assume $\left(\omega^{\xi}+1\right)^{(\xi)}=\left\{\omega^{\xi}\right\}$ for all $\xi<\alpha$, where $\alpha>1$. If $\beta<\omega^{\alpha}$, then we can write $\beta=\gamma+\omega^{\delta}$, where $\delta<\alpha$ [3, p. 67]. By induction, $\omega^{\delta} \notin\left(\omega^{\delta}+1\right)^{(\alpha)}$, so by 4.3 , $\omega^{\prime} \notin\left(\omega^{\alpha}+1\right)^{(\alpha)}$. Thus, by $4.4, \beta \notin\left(\omega^{\alpha}+1\right)^{(\alpha)}$. Hence, $\left(\omega^{\alpha}+1\right)^{(\alpha)} \subseteq\left\{\omega^{\alpha}\right\}$. If $\alpha$ is a non-limit ordinal, then by $4.3,4.4$ and the induction hypothesis, $\left(\omega^{\alpha}+1\right)^{(\alpha-1)}$ contains $\omega^{\alpha-1} n$ for $n=1,2, \cdots$. Thus, $\left(\omega^{\alpha}+1\right)^{(\alpha)}$ contains $\lim _{n} \omega^{\alpha-1} n=\omega^{\alpha}$. If $\alpha$ is a limit ordinal, and if $\xi \leq \zeta<\alpha$, then by 4.3 and the induction hypothesis $\omega^{\zeta} \in\left(\omega^{\alpha}+1\right)^{(\zeta)} \subseteq\left(\omega^{\alpha}+1\right)^{(\xi)}$. Thus, $\omega^{\alpha}=$ $\lim _{\xi \leq \zeta<\alpha} \omega^{\zeta} \in\left(\omega^{\alpha}+1\right)^{(\xi)}$ and therefore $\omega^{\alpha} \in \cap_{\xi<\alpha}\left(\omega^{\alpha}+1\right)^{(\xi)}=\left(\omega^{\alpha}+1\right)^{(\alpha)}$. Hence $\left(\omega^{\alpha}+1\right)^{(\alpha)}=\left\{\omega^{\alpha}\right\}$, and the induction is complete.

THEOREm 4.6. Let $B$ be an infinite Boolean algebra with a well ordered basis, let $\alpha$ be an ordinal greater than zero and let $n$ be a positive integer. Then the following conditions are equivalent:

(i) $B$ has an ordered basis of type $\omega^{\alpha} n+1$;

(ii) $X(B)$ is homeomorphic to $\omega^{\alpha} n+1$;

(iii) $X(B)^{(\alpha)}$ contains precisely $n$ points.

Proof. Because of 2.5, 2.8 and 4.2, it suffices to show that $\left(\omega^{\alpha} n+1\right)^{(\alpha)}$ contains precisely $n$ points. This fact follows from 4.5 and 3.2 (vi) since $\omega^{\alpha} n+1$ is the disjoint union of the open intervals $\left(\omega^{\alpha}\right],\left[\omega^{\alpha}+1, \omega^{\alpha} 2\right], \cdots$, $\left[\omega^{\alpha}(n-1)+1, \omega^{\alpha} n\right]$, each of which is homeomorphic to $\omega^{\alpha}+1$.

5. The free product decomposition of Boolean algebras with well ordered bases. If $B_{1}$ and $B_{2}$ are Boolean algebras, then the product space $X\left(B_{1}\right) \times X\left(B_{2}\right)$ is compact and totally disconnected. The Boolean algebra $B$ of open-and-closed subsets of $X\left(B_{1}\right) \times X\left(B_{2}\right)$ is called the free product of $B_{1}$ and $B_{2}$ and is denoted $B_{1} * B_{2}$. We are going to apply the results of the preceding section to determine the ways in which a Boolean algebra with a well ordered basis can be written as a free product.

Since $X\left(B_{1}\right) \times X\left(B_{2}\right)$ contains closed subspaces homeomorphic to $X\left(B_{1}\right)$, for example $X\left(B_{1}\right) \times\{p\}$ where $p$ is any point of $X\left(B_{2}\right)$, the duality theory implies that if $B=B_{1} * B_{2}$, then $B_{1}$ is a homomorphic image of $B$. By symmetry, so is $B_{2}$. Thus by [6, Theorem 2.2]:

Lemma 5.1. If $B$ is a Boolean algebra with a well ordered basis, and if $B=B_{1} * B_{2}$, then $B_{1}$ and $B_{2}$ are Boolean algebras with well ordered 
bases.

The converse of this lemma is not generally true. In fact, the free product of two Boolean algebras with well ordered bases need not even have an ordered basis.

LEmma 5.2. Let $\omega_{1}$ be the first uncountable ordinal. Then the Boolean algebra $B\left(\omega_{1}+1\right) * B(\omega+1)$ does not have an ordered basis.

Proof. The Boolean space of $B\left(\omega_{1}+1\right) * B(\omega+1)$ is homeomorphic to $X=\left(\omega_{1}+1\right) \times(\omega+1)$. We will prove that if $X$ is homeomorphic to a chain, then the same is true of $X-\left\{\left\langle\omega_{1}, \omega\right\rangle\right\}$ (in the relative topology). But $X-\left\{\left\langle\omega_{1}, \omega\right\rangle\right\}$ is not normal [8], whereas every topological chain is normal.

First observe that if $C$ is a chain and $x \in C$ is a point that neither covers, nor is covered (and is not an end point), then the relative topology of $C-\{x\}$ coincides with its interval topology. To see this, note that since $C-\{x\}$ is open in $C$, every open interval of $C-\{x\}$ is open in $C$. On the other hand, if $I$ is an open interval of $C$ and if neither end-point of $I$ is $x$, then $I \cap(C-\{x\})$ is an open interval of $C-\{x\}$. If $x$ is one end-point of $I$, say the lower one, then $I \cap(C-\{x\})=$ $\mathrm{U}_{y>x}(I \cap\{z \in C \mid z>y\})$, since $x$ is not covered. Thus $I \cap(C-\{x\})$ is open in this case also. Now suppose that $X$ is homeomorphic to a chain $C$. Then the point $x \in C$ which corresponds to $\left\langle\omega_{1}, \omega\right\rangle$ is the limit of a sequence of type $\omega$ because $\left\langle\omega_{1}, \omega\right\rangle$ is the limit of $\left\{\left\langle\omega_{1}, 1\right\rangle,\left\langle\omega_{1}, 2\right\rangle, \cdots\right\}$ in $X$. If $x$ is either covered, covers, or is an end-point in $C$, then $C$ satisfies the first countability axiom at $x$, contrary to the fact that $X$ does not have a countable neighborhood basis at $\left\langle\omega_{1}, \omega\right\rangle$. The alternative, by the preceding paragraph, is that $C-\{x\}$ (and hence $X-\left\{\left\langle\omega_{1}, \omega\right\rangle\right\}$ ) is normal in the relative topology. Thus, $X$ cannot be homeomorphic to a chain.

THEOREM 5.3. Let $B_{1}$ and $B_{2}$ be infinite Boolean algebras with well ordered bases. If $B_{1}$ is uncountable, then $B_{1} * B_{2}$ is a Boolean algebra which does not have an ordered basis.

Proof. By 2.9, $X\left(B_{1}\right)$ and $X\left(B_{2}\right)$ are homeomorphic to infinite nonlimit ordinals $\kappa_{1}$ and $\kappa_{2}$ respectively. Moreover, $\kappa_{1}$ is uncountable, since otherwise $B_{1}$ would have a countable ordered basis and would therefore be countable. Thus, $X\left(B_{1}\right)$ contains a closed subspace homeomorphic to $\omega_{1}+1$ and $X\left(B_{2}\right)$ contains a closed subspace homeomorphic to $\omega+1$. Consequently $X\left(B_{1}\right) \times X\left(B_{2}\right)$ contains a closed subspace homeomorphic to $\left(\omega_{1}+1\right) \times(\omega+1)$. Thus, $X\left(B_{1}\right) \times X\left(B_{2}\right)$ cannot be homeomorphic to a chain, since otherwise, by 3.6, $\left(\omega_{1}+1\right) \times(\omega+1)$ would be homeomorphic to a chain, contrary to 5.2 . 
CoRollary 5.4. If $\alpha$ is an uncountable ordinal, and if $B\left(\omega^{\alpha} n+1\right)$ isomorphic to $B_{1} * B_{2}$, then either $B_{1}$ or $B_{2}$ is finite.

CoRollary 5.5. If $B_{1}$ and $B_{2}$ are infinite Boolean algebras with 'well ordered bases, then the following conditions are equivalent:

(i) $B_{1} * B_{2}$ has a well ordered basis;

(ii) $B_{1} * B_{2}$ has an ordered basis;

(iii) $B_{1}$ and $B_{2}$ are countable.

Proof. Clearly (i) implies (ii) and (ii) implies (iii) by 5.3. Suppose $B_{1}$ and $B_{2}$ are countable. By $2.9, X\left(B_{1}\right)$ and $X\left(B_{2}\right)$ are countable. Thus $X\left(B_{1}\right) \times X\left(B_{2}\right)$ is countable, so $X\left(B_{1}\right) \times X\left(B_{2}\right)$ is homeomorphic to an ordinal [5, Theorem 1].

The free product of a Boolean algebra $B_{1}$ with an ordered basis with a finite Boolean algebra $B_{2}$ has an ordered basis. For $X\left(B_{2}\right)$ is finite, so it can be ordered arbitrarily, and the order topology determined by the lexicographic ordering of $X\left(B_{2}\right) \times X\left(B_{1}\right)$ agrees with the product topopology. Indeed in the lexicographic ordering $X\left(B_{2}\right) \times X\left(B_{1}\right)$ is a finite union of open-and-closed intervals, each homeomorphic to $X\left(B_{1}\right)$. Moreover, if $X\left(B_{1}\right)$ is well ordered, so is the product space. Using the results of section four, it is easy to determine $B_{1} * B_{2}$ when $B_{2}$ is finite and $B_{1}$ is infinite and has a well ordered basis. Let $B_{1}$ have an ordered basis of type $\omega^{\alpha} m+1$ with $\alpha>0, m>0$, and let $B_{2}$ contain $n$ atoms. By 3.2 (vi), $\left(X\left(B_{1}\right) \times X\left(B_{2}\right)\right)^{(\alpha)}$ is the disjoint union of $n$ copies of $X\left(B_{1}\right)^{(\alpha)}$, and therefore consists of $m n$ points. Thus by 4.6 ,

$$
B\left(\omega^{\alpha} m+1\right) * B(n) \cong B\left(\omega^{\alpha} m n+1\right) .
$$

THEOREM 5.6. If $B$ is an infinite Boolean algebra with a well ordered basis, then $B$ is isomorphic to a unique free product $B_{1} * B_{2}$, where $B_{1}=B\left(\omega^{\alpha}+1\right)$ for some ordinal $\alpha>0$ and $B_{2}=B(n)$ for some integer $n \geq 1$.

If $B$ is any Boolean algebra, then $B * B(1)$ is isomorphic to $B$. We shall call $B$ indecomposable if this is the only possible free product decomposition of $B$. With this definition, 5.4 and 5.6 give an interesting result.

CoRollaRY 5.7. If $\alpha$ is an uncountable ordinal, then $B\left(\omega^{\alpha}+1\right)$ is indecomposable.

The free product factorization of $B(n)$ runs entirely parallel to the factorization of $n$ into prime integers. Thus 5.6 and 5.7 settle the problem of unique factorization of uncontable Boolean algebras with well ordered bases and it only remains to consider algebras $B\left(\omega^{\alpha}+1\right)$, where $\alpha$ is a countable ordinal. 
Let $\alpha=\omega^{\lambda_{1}} m_{1}+\cdots+\omega^{\lambda_{k}} m_{k}$ and $\beta=\omega^{\lambda_{1}} n_{1}+\cdots+\omega^{\lambda_{k}} n_{k}$, where $\lambda_{1}>\cdots>\lambda_{k}$ and the $m_{i}$ and $n_{i}$ are non-negative integers. The natural sum [4, section 75] of $\alpha$ and $\beta$ is defined to be

$$
\alpha \# \beta=\omega^{\lambda_{1}}\left(m_{1}+n_{1}\right)+\cdots+\omega^{\lambda_{k}}\left(m_{k}+n_{k}\right) .
$$

Note that $\alpha \# \beta=\beta \# \alpha, \alpha \#(\beta \# \gamma)=(\alpha \# \beta) \# \gamma$ and if $\alpha \leq \beta, \gamma \leq \delta$, then $\alpha \# \gamma \leq \beta \# \delta$ with equality only if $\alpha=\beta$ and $\gamma=\delta$.

THEOREM 5.9. If $\alpha$ and $\beta$ are countable ordinals greater than zero, then $B\left(\omega^{\alpha}+1\right) * B\left(\omega^{\beta}+1\right)$ is isomorphic to $B\left(\omega^{\alpha \sharp \beta}+1\right)$.

Proof. Assume inductively that the theorem is true for all pairs $\langle\gamma, \delta\rangle$ such that $\gamma \# \delta<\alpha \# \beta$. Without loss of generality, assume $\alpha \geq \beta$. Let $\alpha=\omega^{\lambda} n+\gamma$, where $\gamma<\omega^{\lambda} \leq \alpha$. If $\gamma>0$, the induction hypothesis gives isomorphisms

$$
\begin{aligned}
B\left(\omega^{\alpha}+1\right) * B\left(\omega^{\beta}+1\right) & \cong B\left(\omega^{\omega^{\lambda} n}+1\right) * B\left(\omega^{\gamma}+1\right) * B\left(\omega^{\beta}+1\right) \\
& \cong B\left(\omega^{\omega^{\lambda} n}+1\right) * B\left(\omega^{\gamma \# \beta}+1\right) .
\end{aligned}
$$

Thus, we may suppose that $\alpha=\omega^{\lambda} n$ and in particular $\alpha \# \beta=\alpha+\beta$. Under this assumption, we can complete the proof by showing that $\left(\left(\omega^{\alpha}+1\right) \times\left(\omega^{\beta}+1\right)\right)^{(\alpha+\beta)}=\left\{\left\langle\omega^{\alpha}, \omega^{\beta}\right\rangle\right\}$.

By $4.5,\left\langle\omega^{\alpha}, \xi\right\rangle \in\left(\left(\omega^{\alpha}+1\right) \times\left(\omega^{\beta}+1\right)\right)^{(\alpha)}$ for all $\xi \leq \omega^{\beta}$. Hence, $\left\langle\omega^{\alpha}, \omega^{\beta}\right\rangle \in\left(\left(\omega^{\alpha}+1\right) \times\left(\omega^{\beta}+1\right)\right)^{(\alpha+\beta)}$ by 4.5 again. Suppose that $\langle\xi, \eta\rangle$ is a point of $\left(\omega^{\alpha}+1\right) \times\left(\omega^{\beta}+1\right)$ which is distinct from $\left\langle\omega^{\alpha}, \omega^{\beta}\right\rangle$. Let $\xi=\xi_{1}+\omega^{\mu}, \eta=\eta_{1}+\omega^{\nu}$. Then $\mu \# \nu<\alpha \# \beta$, since either $\xi<\omega^{\alpha}$ or $\eta<\omega^{\beta}$. Define $A=\left\{\langle\rho, \sigma\rangle \mid \xi_{1}<\rho \leq \xi, \eta_{1}<\sigma \leq \eta\right\}$. Then $A$ is an open subset of $\left(\omega^{\alpha}+1\right) \times\left(\omega^{\beta}+1\right)$ which is homeomorphic to $\left(\omega^{\mu}+1\right) \times\left(\omega^{\nu}+1\right)$ if $\mu>0$ and $\nu>0$, to $\omega^{\nu}+1$ if $\mu=0, \nu>0$, to $\omega^{\mu}+1$ if $\mu>0, \nu=0$, and to a one point space if $\mu=\nu=0$. By the induction hypothesis in case $\mu>0$ and $\nu>0$, and by 4.5 in the other cases, $A^{(\alpha+\beta)}=A^{(\alpha \# \beta)}=\phi$. But $A$ is open, so by 3.2 (vi), $A^{(\alpha+\beta)}=A \cap\left(\left(\omega^{\alpha}+1\right) \times\left(\omega^{\beta}+1\right)\right)^{(\alpha+\beta)}$. Therefore, $\langle\xi, \eta\rangle \notin\left(\left(\omega^{\alpha}+1\right) \times\left(\omega^{\beta}+1\right)\right)^{(\alpha+\beta)}$. Thus,

$$
\left(\left(\omega^{\alpha}+1\right) \times\left(\omega^{\beta}+1\right)\right)^{(\alpha+\beta)}=\left\{\left\langle\omega^{\alpha}, \omega^{\beta}\right\rangle\right\} .
$$

CoROLlaRY 5.10. Let $\alpha$ be a countable ordinal greater than zero. Then $B\left(\omega^{\alpha}+1\right)$ is indecomposable if and only if $\alpha$ is of the form $\omega^{\beta}$ for some (countable) ordinal $\beta$.

COROLlary 5.11. Let $\alpha$ be a countable ordinal greater than zero and suppose $\alpha=\omega^{\lambda_{1}} m_{1}+\cdots+\omega^{\lambda_{k}} m_{k}$ with $\lambda_{1}>\cdots>\lambda_{k}$ and $m_{i}>0$. Let

$$
B_{i}=B\left(\omega^{\omega^{\lambda} i}+1\right)
$$


Then

$$
B\left(\omega^{\alpha}+1\right) \cong B_{1}^{m_{1}} * \cdots * B_{k}^{m_{k}},
$$

where $B_{i}^{m_{i}}$ is the free product of $B_{i}$ with itself $m_{i}$ times, and this decomposition into indecomposables is unique up to the order of the factors.

\section{BIBLIOGRAPHY}

1. G. Birkhoff, Lattice Theory, 2nd Ed., Amer. Math. Soc. Colloquium Publ. 25, New York, 1948.

2. F. Hausdorff, Grundzüge der Mengenlehre, Leipzig, 1914.

3. - Mengenlehre, Leipzig, 1927.

4. G. Hessenberg, Grundbegriffe der Mengenlehre, Gottingen, 1906.

5. S. Mazurkiewicz and W. Sierpinski, Contribution à la topologie des ensembles dénombrables, Fund. Math. 1 (1920), 17-27.

6. A. Mostowski and A. Tarski, Boolesche Ringe mit geordneter Basis, Fund. Math., 32 (1939), 69-86.

7. M. H. Stone, Applications of the theory of Boolean rings to general topology, Trans. Amer. Math. Soc., 41 (1937), 375-481.

7. A. Tychonoff, Über die topologische Erwerterung von Raumen, Math. Ann., 102 (1929), 544-561.

IDAHO"STATE:COLLEGE

UNIVERSITY OF WASHINGTON 


\section{PACIFIC JOURNAL OF MATHEMATICS}

\section{EDITORS}

\author{
David Gilbarg \\ Stanford University \\ Stanford, California \\ F. H. Brownell \\ University of Washington \\ Seattle 5 , Washington
}

\author{
A. L. Whiteman \\ University of Southern California \\ Los Angeles 7, California \\ L. J. PAIGE \\ University of California \\ Los Angeles 24, California
}

\section{ASSOCIATE EDITORS}

\author{
E. F. BECKENBACH \\ T. M. CHERRY \\ D. DERRY
}
E. HEWITT
A. HORN
L. NACHBIN
M. OHTSUKA
H. L. ROYDEN
M. M. SCHIFFER

E. SPANIER

E. G. STRAUS

F. WOLF

\section{SUPPORTING INSTITUTIONS}

\author{
UNIVERSITY OF BRITISH COLUMBIA \\ CALIFORNIA INSTITUTE OF TECHNOLOGY \\ UNIVERSITY OF CALIFORNIA \\ MONTANA STATE UNIVERSITY \\ UNIVERSITY OF NEVADA \\ NEW MEXICO STATE UNIVERSITY \\ OREGON STATE COLLEGE \\ UNIVERSITY OF OREGON \\ OSAKA UNIVERSITY \\ UNIVERSITY OF SOUTHERN CALIFORNIA
}

\author{
STANFORD UNIVERSITY \\ UNIVERSITY OF TOKYO \\ UNIVERSITY OF UTAH \\ WASHINGTON STATE COLLEGE \\ UNIVERSITY OF WASHINGTON

AMERICAN MATHEMATICAL SOCIETY
CALIFORNIA RESEARCH CORPORATION
HUGHES AIRCRAFT COMPANY
SPACE TECHNOLOGY LABORATORIES
NAVAL ORDNANCE TEST STATION

Mathematical papers intended for publication in the Pacific Journal of Mathematics should be typewritten (double spaced), and the author should keep a complete copy. Manuscripts may be sent to any one of the four editors. All other communications to the editors should be addressed to the managing editor, L. J. Paige at the University of California, Los Angeles 24, California. ..

50 reprints per author of each article are furnished free of charge; additional copies may be obtained at cost in multiples of 50 .

The Pacific Journal of Mathematics is published quarterly, in March, June, September, and December. The price per volume (4 numbers) is $\$ 12.00$; single issues, $\$ 3.50$. Back numbers are available. Special price to individual faculty members of supporting institutions and to individual members of the American Mathematical Society: $\$ 4.00$ per volume; single issues, $\$ 1.25$.

Subscriptions, orders for back numbers, and changes of address should be sent to Pacific Journal of Mathematics, 2120 Oxford Street, Berkeley 4, California.

Printed at Kokusai Bunken Insatsusha (International Academic Printing Co., Ltd.), No. $6_{4}$ 2-chome, Fujimi-cho, Chiyoda-ku, Tokyo, Japan.

PUBLISHED BY PACIFIC JOURNAL OF MATHEMATICS, A NON-PROFIT CORPORATION

The Supporting Institutions listed above contribute to the cost of publication of this Journăl, but they are not owners or publishers and have no responsibility for its content or policies. 


\section{Pacific Journal of Mathematics}

\section{Vol. 10, No. $3 \quad$ November, 1960}

Glen Earl Baxter, An analytic problem whose solution follows from a simple

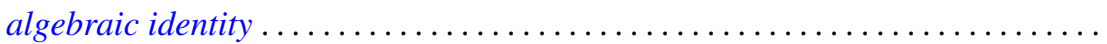

Leonard D. Berkovitz and Melvin Dresher, A multimove infinite game with linear payoff. .

Earl Robert Berkson, Sequel to a paper of A. E. Taylor ......................

Gerald Berman and Robert Jerome Silverman, Embedding of algebraic systems.... 767

Peter Crawley, Lattices whose congruences form a boolean algebra . . . . . ...... 777

Robert E. Edwards, Integral bases in inductive limit spaces . . . . . . . . . . . . . . .

Daniel T. Finkbeiner, II, Irreducible congruence relations on lattices . . . . . . . . . .

William James Firey, Isoperimetric ratios of Reuleaux polygons . . . . . . . . . . . 787

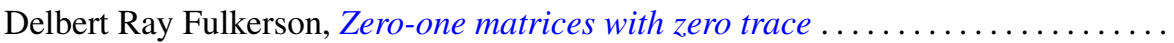

Leon W. Green, A sphere characterization related to Blaschke's conjecture........

Israel (Yitzchak) Nathan Herstein and Erwin Kleinfeld, Lie mappings in

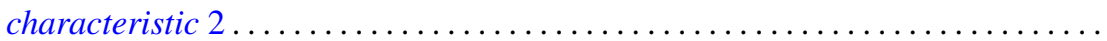

Charles Ray Hobby, A characteristic subgroup of a $p$-group .................

R. K. Juberg, On the Dirichlet problem for certain higher order parabolic

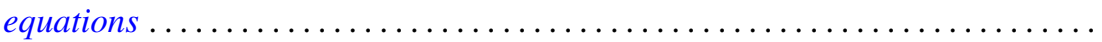

Melvin Katz, Infinitely repeatable games ......................

Emma Lehmer, On Jacobi functions . . . . . . . . . . . . . . . . . . . . . . . . .

D. H. Lehmer, Power character matrices

Henry B. Mann, A refinement of the fundamental theorem on the density of the sum of two sets of integers.

Marvin David Marcus and Roy Westwick, Linear maps on skew symmetric matrices: the invariance of elementary symmetric functions . .

Richard Dean Mayer and Richard Scott Pierce, Boolean algebras with ordered

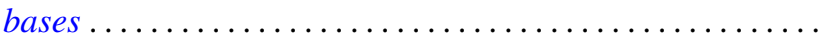

Trevor James McMinn, On the line segments of a convex surface in $E_{3} \ldots$

Frank Albert Raymond, The end point compactification of manifolds ..

Edgar Reich and S. E. Warschawski, On canonical conformal maps of regions of arbitrary connectivity

Marvin Rosenblum, The absolute continuity of Toeplitz's matrices...

Lee Albert Rubel, Maximal means and Tauberian theorems . .

Helmut Heinrich Schaefer, Some spectral properties of positive linear operators

Jeremiah Milton Stark, Minimum problems in the theory of pseudo-conformal transformations and their application to estimation of the curvature of the invariant metric.

Robert Steinberg, The simplicity of certain groups ...

Hisahiro Tamano, On paracompactness. .

Angus E. Taylor, Mittag-Leffler expansions and spectral theory .

Marion Franklin Tinsley, Permanents of cyclic matrices ...... . 